\title{
Efficient VLSI Implementation of the C-MANTEC Conn Algorithm by Using PID Controllers
}

\author{
J. Jeya Caleb ${ }^{1 *}$, M. Kannan ${ }^{2}$ \\ ${ }^{1}$ Department of ECE, Saveetha Engineering College, Chennai, India \\ ${ }^{2}$ Department of ECE, MIT Campus, Anna University, Chennai, India \\ Email: *jeycaleb@gmail.com
}

How to cite this paper: Caleb, J.J. and Kannan, M. (2017) Efficient VLSI Implementation of the C-MANTEC Conn Algorithm by Using PID Controllers. Circuits and Systems, 8, 253-260.

https://doi.org/10.4236/cs.2017.811018

Received: May 8, 2016

Accepted: May 16, 2016

Published: November 3, 2017

Copyright ( 92017 by authors and Scientific Research Publishing Inc. This work is licensed under the Creative Commons Attribution International License (CC BY 4.0).

http://creativecommons.org/licenses/by/4.0/

(c) (i) Open Access

\begin{abstract}
Through the research on the existing C-MANTEC neural network and PID control technology, this paper presents an improved C-MANTEC algorithm based on PID control system. The combining of the artificial neural networks with conventional PID control helps in exploring their respective advantages to forming the intelligent PID control. From UCI Repository cancer dataset, the developed system is tested. The results show that the scheme can not only improve the speed of the algorithm in the training process but also improve the generalization capability of the network, which further enhances the performance of PID controllers. The overall power consumed is also reduced to a greater extent.
\end{abstract}

\section{Keywords}

C-MANTEC, PID, Generalization Capability, Cancer, Intelligent Control

\section{Introduction}

One of the principal causes of recent deaths is due to the severe complication of Cancer. Though it is a non-communicable disease, it needs an early diagnosis because the abnormal cancer cells spread quickly. Malignant neoplasm describes Cancerous cells. They demonstrate uncontrolled cell malignant neoplasm. The non-cancerous tumors are called benign tumors. Mammography, MRI, CT, endoscopy, ultrasonography, nuclear medicine imaging and positron emission tomography are some of the methods to diagnose Cancer.

Early diagnosis requires correct and reliable diagnosis procedure that will allow physicians to distinguish benign tumors from malignant ones. An accurate and effective diagnosis method is vital, and that can be treated as a machine learning problem. This problem can be solved using neural networks. 
Artificial Neural Network acts as an emulator of the biological neural system. In the naturally inspired system, the electrical signals are modulated at the synapse which can be imbibed to build the neural schema. This learning is interpreted as weight adjustments among the neurons in the neural systems, which can be applied to all non-linear problems such as prediction, system identification, pattern recognition, and control. Therefore, they can be proudly called as universal mappers.

In this paper, a novel neural network algorithm combining the recently proposed C-Mantec Algorithm with PID controllers has been implemented to detect the presence of cancer. Section 2 deals with the literature survey; Section 3 deals with the proposed methodology whereas Section 4 deals with outcomes and discussion. Finally, Section 5 deals with a conclusion.

\section{Literature Survey}

In [1] C-Mantec CoNN model was introduced. Effectual area utilization and delay minimization with 47 times increase of the speed factor was focused. The frequency level of $72.72 \mathrm{MHz}$ was achieved by the utilized hardware. This work couldn't nullify the error. Giap Hoang Nguyen et al., [2] have propounded an auto-tuning of the PID controller based on the RBF neural network and relay feedback approach. They were able to achieve a frequency of $117 \mathrm{~Hz}$. It was not able to build an optimized system with the best performance in the time since the iterations don't converge so easily, though more routing ability was revealed by the work.

In [3] the authors have reviewed the then state-of-the-art neural algorithms and have analyzed about various algorithms. Though Back Propagation could limit the errors to required levels, it fails to achieve generalization ability. They have reported that LM algorithm in spite of being faster had two significant drawbacks. One, it couldn't be applied to SLP and major networks.

Though NBN could overwhelm the demerits of LM algorithm, they were not up to the mark. RBF faced the problem of over fitting and under fitting. The layer density is more when getting into Fully Connected Cascade structures though they have proven to be better than MLPs. Overall, when the intensity of the process of nonlinear mapping is increased the above networks fall short in noise and accuracy.

In [4], the efficiency of C-Mantec attained better performance nearly $97 \%$ in the rule-based programming. Architecture with less number of layers was generated manifesting powerful computational capability and flexibility. Although it outperformed concerning computation capability, it couldn't concentrate on error detection. The work reported in [5] recommended, less utilization of sources (low cost FPGAs) along with less delay concentration. They couldn't optimize power. A PID controller [6] with area-efficient DA-based algorithm was introduced resulting in less delay, low cost and low power dissipation saving $80 \%$ in area and $40 \%$ in power. 
Artificial Neural Network [7] obtained stability in all the four quadrants of operation. In [8], PID controllers have been blended with Back Propagation to attain good performance in adaptivity. The demerit of their work was easy to fall into local minimum and slow convergence. Genetic Algorithm [9] has collaborated with NN based PID for better speed performance. Despite the method developed in [10] could achieve compact implementation of the architecture it wasn't able to cover all the activation functions. Also, this algorithm isn't flexible as needed.

A single PID controller [11] to achieve multi-channel efficiency was introduced which consumed power of $3.66 \mathrm{~mW}$. A system with less cost, less power, and increased speed had been reached. Transductive SVM [12] helps out dimensionality reduction that had been concentrated for better accuracy with fewer data size. Cmantec could perform 17 times better than Artificial Neural Network in execution time [13].

The TSVM was flexible [14] which was built-in directly for transductive learning. Here accuracy could not be achieved and struggled in the choice of Threshold value. PID learning algorithm can also obtain optimized convergence by utilizing LMI techniques [15].

\section{Proposed Methodology}

The procedure followed in designing the architecture for the chosen problem is that four input neurons with no computational capability were taken into account. Then the iterations continued with an input value taken from the cancer dataset obtained from UCI Repository. The iterations continued till the weight value was finalized. Model weight calculation is shown in Table 1 . The hidden layer was first included with a single neuron and the process continued till the growing factor exceeding thermal co-efficient. Thereby the number of neurons needed to learn the incoming data was found to be 3 in the hidden layer. The output neuron called the majority perceptron was set to be one. In this manner, the architecture is constructed, and the neurons learn the incoming data. The implemented architecture is shown in Figure 1.

\section{Architecture}

The novel methodology used in this paper is the inclusion of PID block with the

Table 1. Weight calculation.

\begin{tabular}{cccccccc}
\hline $\begin{array}{c}\text { INPUT } \\
(\mathbf{x i})\end{array}$ & $\begin{array}{c}\text { WEIGHT } \\
(\mathrm{W})\end{array}$ & $\begin{array}{c}\text { NET } \\
(\mathbf{x i W})\end{array}$ & $\begin{array}{c}\text { THERESHOLD } \\
(\mathbf{0 . 5})\end{array}$ & $\begin{array}{c}\text { OUT } \\
(\mathrm{S})\end{array}$ & $\mathrm{T}=\mathrm{T}_{0}-\mathrm{MI}$ & $\begin{array}{c}\mathrm{T}_{\mathrm{fac}}=\mathrm{T} / \mathrm{T}_{0} \\
\exp \{\Phi / \mathrm{T}\}\end{array}$ & $\begin{array}{c}\Delta_{\mathrm{W}}= \\
(\mathrm{t}-\mathrm{s}) \mathbf{x}_{\mathrm{i}} \mathrm{T}_{\mathrm{fac}}\end{array}$ \\
\hline 0.645 & 0 & 0 & $0(<0.5)$ & 0 & 9.9 & 1.2116 & 0.78 \\
0.645 & 0.78 & 0.45 & $0.45(<0.5)$ & 0 & 9.8 & 1.2018 & 0.77 \\
0.645 & 0.77 & 0.49 & $0.49(<0.5)$ & 0 & 9.7 & 1.1921 & 0.76 \\
0.645 & 0.76 & 0.49 & $0.49(<0.5)$ & 0 & 9.6 & 1.1182 & 0.76 \\
\hline
\end{tabular}


Neural Network block. The conventional PID controllers have been superseded by PID controllers based on Neural Network in reducing the error between the output of the system and the expected value. The structure of PID control system based on C-MANTEC neural network is shown in Figure 2. The kp, kd and ki parameters of the PID controller are adjusted based on the system's operational status, and thereby the output neurons decide those values. The weight coefficients are calculated using the C-MANTEC algorithm as already illustrated.

The Finite State Machine generates the required control signals. It controls the overall operations of the network. Firstly, it activates the input neurons to load the signals of the neurons. Then in three clock cycles, the hidden layer is activated. Then the output is generated for five clock cycles. The out signals are then generated by the state machine. The FSM is designed using VHDL to suit to different configurations flexibly. The inputs are taken from the database which is then copied in a notepad and in turn extracted using the "file" concept in VHDL. The FSM generates the weight ROM addresses to be used by all neurons.

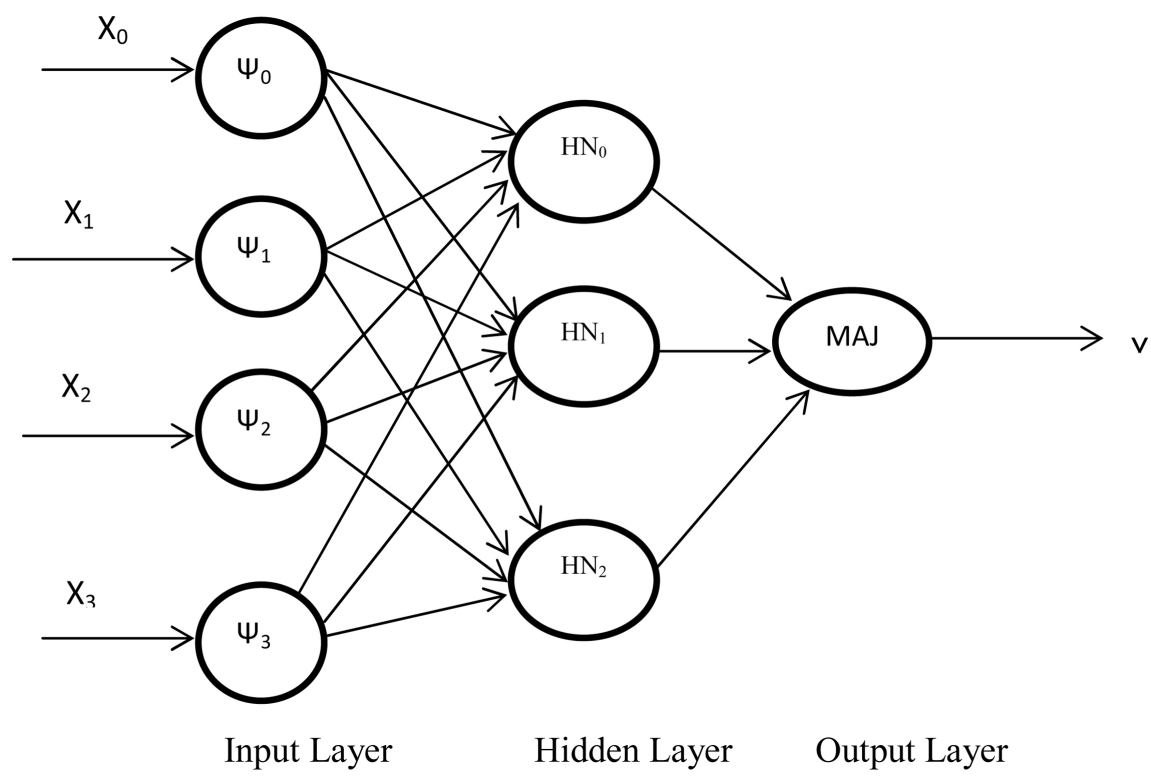

Figure 1. Implemented architecture of C-MANTEC network.

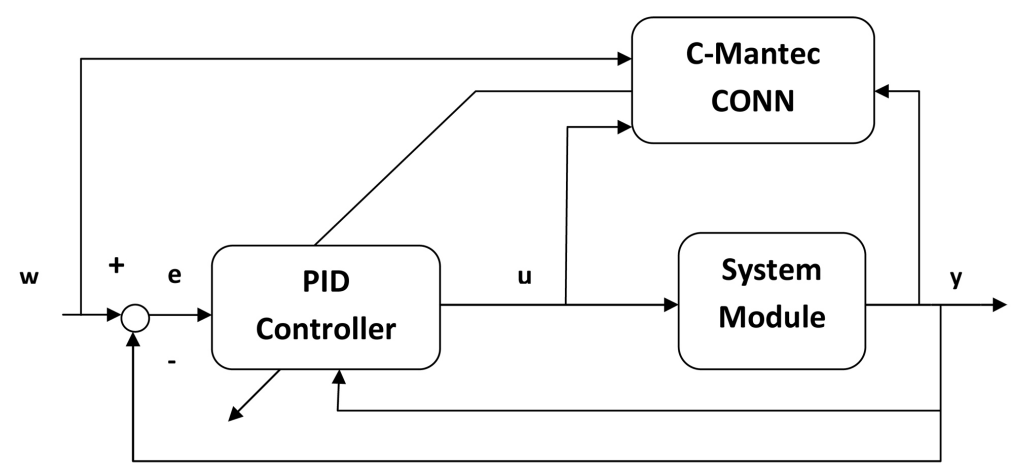

Figure 2. Structure of the C-Mantec CONN using PID Controller. 
Once the propagation phase starts, the network's output is put on the show. The block diagram of the network is shown in Figure 3.

In the neuron architecture, multiply and accumulate structure are used. In this structure, there is one multiplier and one accumulator per neuron. The data from previous layers enter into next layers serially. Then are multiplied with their corresponding weights and summed in the accumulator. They are all synchronized by the clock signals. The structure of the RAM used in this architecture is shown in Figure 4. The number of clock signals depends on the number of the interconnections in the previous layer. This neuron structure is shown in Figure 5.

In this paper, for the defects of general C-MANTEC algorithm such as slow

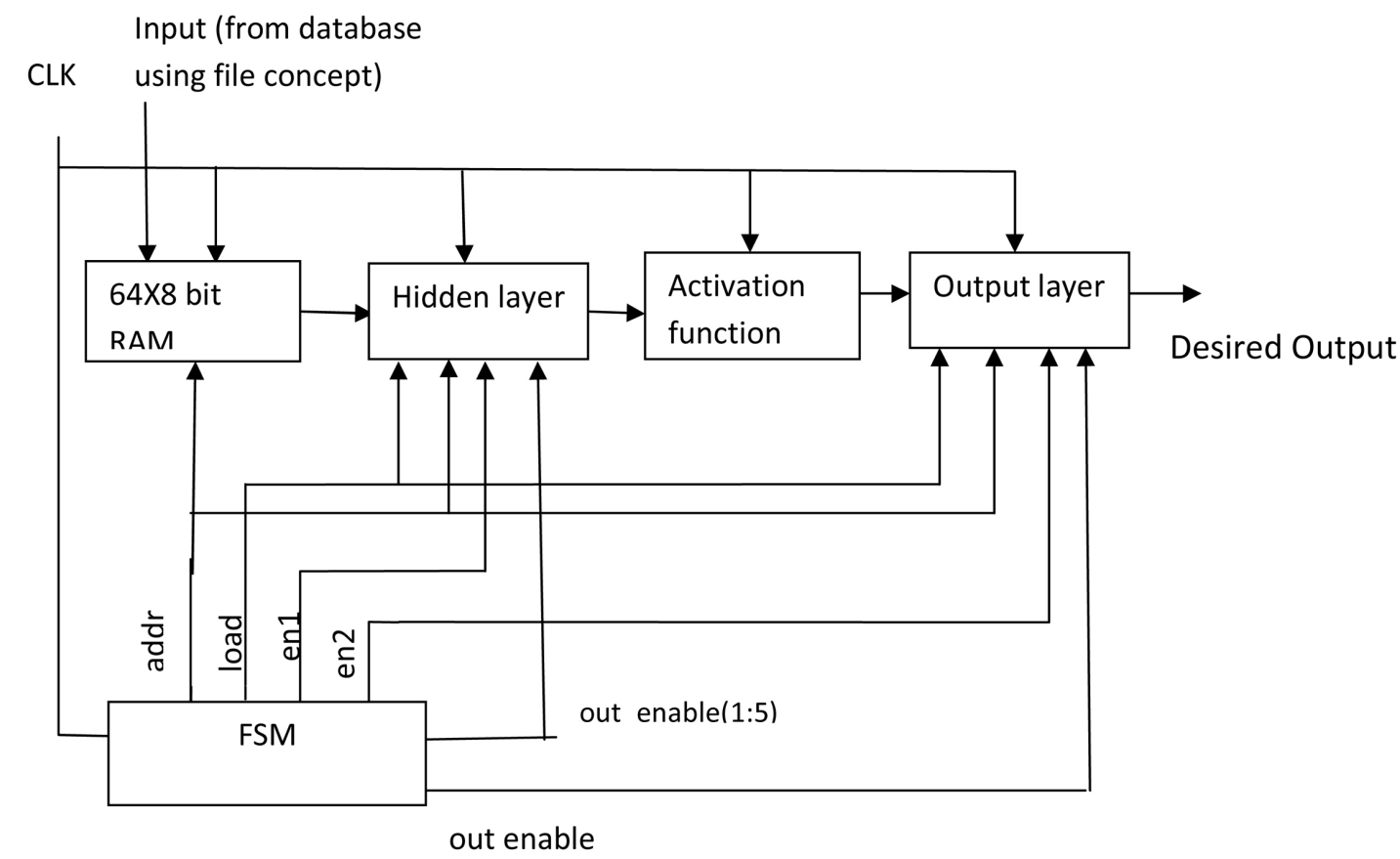

Figure 3. Neural network model.

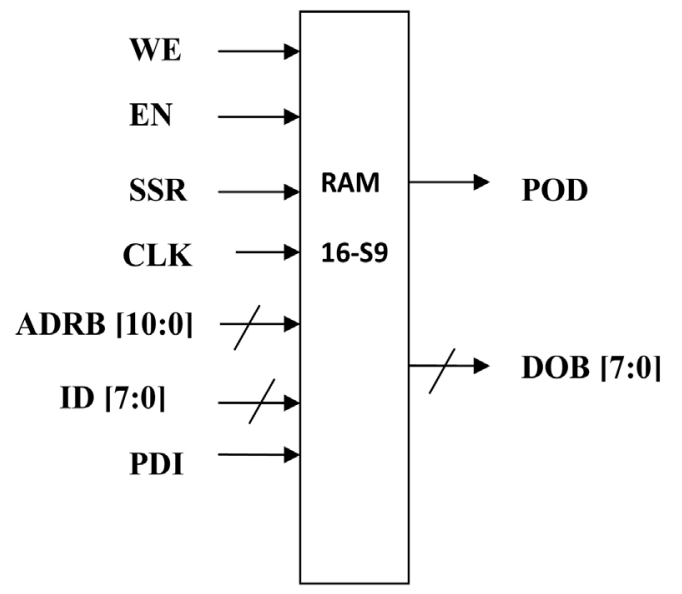

\begin{tabular}{|l|c|c|}
\hline Description of Signals & Terms & I/O \\
\hline Input Data Bus & ID & Input \\
\hline Parity Data Input Bus & PDI & Input \\
\hline Data Output Bus & DOB & Output \\
\hline Parity Output Data & POD & Output \\
\hline Address Bus & ADRB & Input \\
\hline Write Enable & WE & Input \\
\hline Clock Enable & EN & Input \\
\hline Synchronous Set/Reset & SSR & Input \\
\hline Clock & CLK & Input \\
\hline
\end{tabular}

Figure $4.64 \times 8$ bit single port RAM. 
convergence an improved C-MANTEC algorithm with less error is proposed, and based on which PID control system's implementation scheme is provided. The results show that the system can improve the algorithm's convergence speed in the training phase, and the trained C-MANTEC network also has efficient adaptive and self-learning capability, which further enhances the performance of PID controllers.

\section{Results \& Discussion}

The Very High Speed HDL has been used to design the system. It was synthesized using Xilinx6.1i and simulated with ModelSim XE III 6.3c. The programming file generated during device programming was downloaded and tested in the IC "XCV400hq240". The Device Utilization summary is presented in Table 2 and in Table 3. the generalization capability is shown.

The over-all output implemented in ModelSim is shown in Figure 6. The network has been designed such that it echoes the presence of cancer whenever an input data is within the specified ranges. The output parameters like delay, total power and frequency are shown in Table 4.

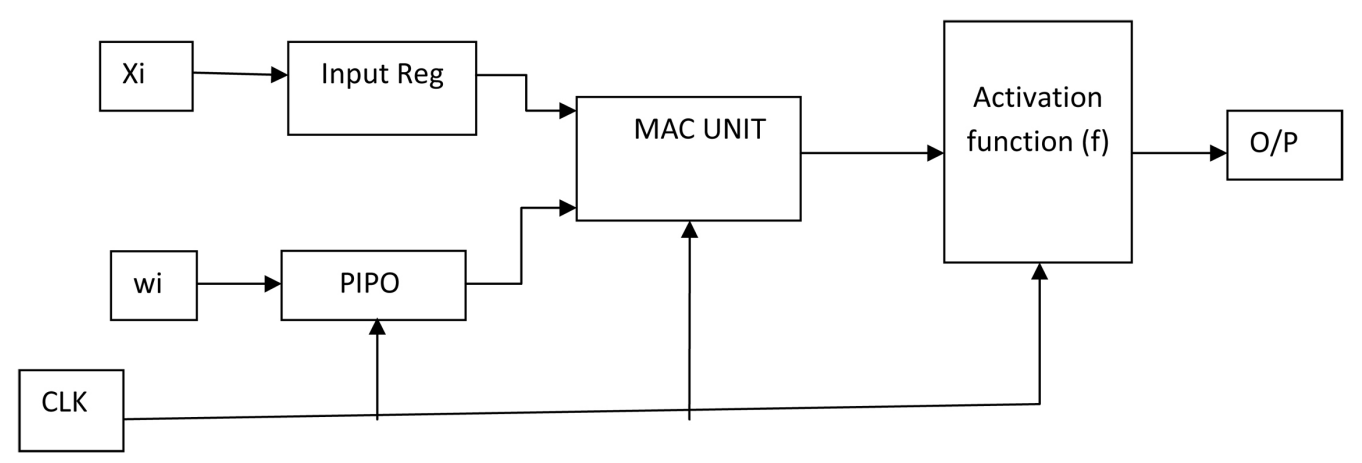

Figure 5. VHDL design of single neuron.

Table 2. Device utilization summary.

\begin{tabular}{cccc}
\hline Logic Utilization & Used & Available & Utilization \\
\hline Number of Slices & 260 & 2448 & $10 \%$ \\
Number of Slice Flip Flops & 254 & 4896 & $5 \%$ \\
Number of 4 input LUTs & 366 & 4896 & $7 \%$ \\
Number of bonded IOBs & 42 & 158 & $26 \%$ \\
Number of MULTI18X18SIOs & 7 & 12 & $58 \%$ \\
Number of GCLKs & 1 & 24 & $4 \%$ \\
\hline
\end{tabular}

Table 3. Generalization capability.

\begin{tabular}{cccc}
\hline FUNCTION & No. of Inputs & \# Neurons & Generalization \\
\hline Cancer & 10 & 7 & $98.1 \pm 1.6$
\end{tabular}


Table 4. Output parameters.

\begin{tabular}{cc}
\hline Parameter & Value \\
\hline Delay & $9.593 \mathrm{~ns}$ \\
Total Power & $34.27 \mathrm{~mW}$ \\
Frequency & $70 \mathrm{MHz}$ \\
\hline
\end{tabular}

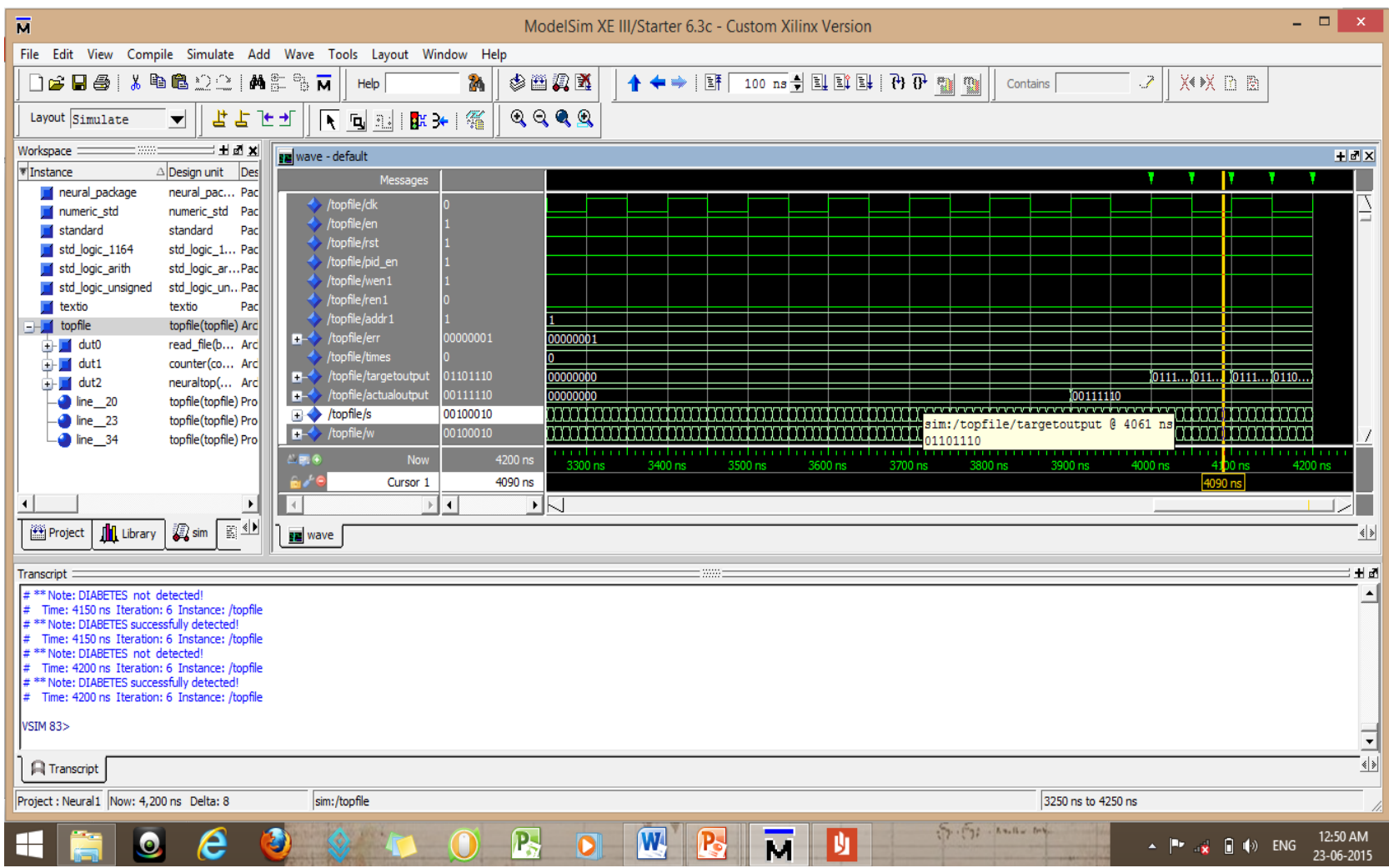

Figure 6. Over-all output.

\section{Conclusion}

It is concluded that the present work demonstrates the appropriateness of the PID controllers using the C-Mantec algorithm for its application in the real-world problems such as cancer detection, robotics, etc., and the algorithm was also synthesized to prove its hardware applicability. It has proved its efficiency and suitability amidst many constraints and therefore can be applied to all the real-time problems.

\section{References}

[1] Ortega-Zamorano, F., Jerez, J.M. and Franco, L. (2014) FPGA Implementation of the C-Mantec Neural Network Constructive Algorithm. IEEE Transactions on Industrial Informatics, 10, 1154-1161. https://doi.org/10.1109/TII.2013.2294137

[2] Nguyen, G.H., Shin, J.-H. and Kim, W.-H. (2015) Autotuning Controller for Motion Control System Based on Intelligent Neural Network and Relay Feedback Approach. IEEE/ASME Transactions on Mechatronics, 20, 1138-1148. https://doi.org/10.1109/TMECH.2014.2344692 
[3] Hunter, D., Yu, H., Pukish, M.S., Kolbusz, J. and Wilamowski, B.M. (2012) Selection of Proper Neural Network Sizes and Architectures-A Comparative Study. IEEE Transactions on Industrial Informatics, 8, 228-240. https://doi.org/10.1109/TII.2012.2187914

[4] Munoz, D.U., Carmona, E.C., Contreras, J.L.S., Franco, L., Torres, L.M.L. and Aragones, J.M.J. (2012) Energy Efficient Programming in WSN Using Constructive Neural Networks. ICIC International Journal of Innovative Computing, Information and Control, 8, 7561-7578.

[5] Himavathi, S., Anitha, D. and Muthuramalingam, A. (2007) Feed Forward Neural Network Implementation in FPGA Using Layer Multiplexing for Effective Resource Utilization. IEEE Transactions on Neural Networks, 18, 880-888. https://doi.org/10.1109/TNN.2007.891626

[6] Chan, Y.F., Moallem, M. and Wang, W. (2007) Design and Implementation of Modular FPGA-Based PID Controllers. IEEE Transactions on Industrial Electronics, 54, 1898-1906. https://doi.org/10.1109/TIE.2007.898283

[7] Maiti, S., Verma, V., Chakraborty, C. and Hori, Y. (2012) An Adaptive Speed Sensorless Induction Motor Drive with Artificial Neural Network for Stability Enhancement. IEEE Transactions on Industrial Informatics, 8, 757-766. https://doi.org/10.1109/TII.2012.2210229

[8] Liu, L. and Luo, J. (2011) Research of PID Control Algorithm Based on Neural Network. Energy Procedia, 13, 6988-6993.

[9] Michiyo, S., Toru, Y. and Toshio, T. (2004) A Design of Neural Net Based PID Controllers with Evolutionary Computation. IEICE Transactions on Fundamentals, E87-A, 2761-2768.

[10] Dinu, A., Cirstea, M.N. and Cirstea, S.E. (2010) Direct Neural-Network Hardware-Implementation Algorithm. IEEE Transactions on Industrial Electronics, 57, 1845-1848. https://doi.org/10.1109/TIE.2009.2033097

[11] Nema, R., Thakur, R. and Gupta, R. (2013) Design \& Implementation of FPGA Based on PID Controller. International Journal of Inventive Engineering and Sciences, 1, 14-16.

[12] Maulik, U., Mukhopadhyay, A. and Chakraborty, D. (2013) Gene-Expression-Based Cancer Subtypes Prediction through Feature Selection and Transductive SVM. IEEE Transactions on Biomedical Engineering, 60, 1111-1117. https://doi.org/10.1109/TBME.2012.2225622

[13] Urda, D., Subirats, J.L., Franco, L. and Jerez, J.M. (2010) Constructive Neural Networks to Predict Breast Cancer Outcome by Using Gene Expression Profiles. IEA/AIE Springer-Verlag, Part I, LNAI 6096, 317-326.

[14] Chakraborty, D. and Das, S. (2012) Cancer Classification through Feature Selection and Transductive SVM using Gene Microarray Data. 3rd International Conference on Emerging Applications of Information Technology, 77-80.

[15] Jing, X. and Cheng, L. (2013) An Optimal PID Control Algorithm for Training Feed forward Neural Networks. IEEE Transactions on Industrial Electronics, 60, 2273-2283. 